NOTA

$321-331$

\title{
DEGLUTIENDO CENTROS Y PERIFERIAS: LA ALTERNATIVA ANTROPOFÁGICA
}

Swallowing centers and peripheries: the antropophagic alternative

Federica Scherbosky*

El Movimiento Antropofágico surge en Brasil, a comienzos de los años 20. El mismo se presenta como un entramado complejo que por un lado articula y condensa el modernismo brasileño, pero que a su vez juega un importante rol en lo que a la crítica artística, política y social respecta. Surge como una posible respuesta, o más bien como un modo de intentar pensar una realidad sumamente compleja por su diversidad característica, con múltiples entrecruzamientos étnicos, religiosos y culturales.

Uno de los hitos fundamentales y que puede considerarse fundacional del mismo es la Semana de Arte Moderno que se organiza en el Teatro Municipal de San Pablo, en 1922. Esta es, sin duda, fruto de un trabajo colectivo, pero quien se halla a la cabeza del movimiento y lo representa en diversas situaciones es Oswald de Andrade, destacado escritor y periodista que nace en San Pablo en 1890 y muere en 1954. De Andrade (2008) considera que la Semana de Arte Moderno marca el inicio de la renovación artística en Brasil y que es la que realmente abre camino para un sinnúmero de cambios culturales.

La idea de la antropofagia se constituye como una respuesta a la necesidad de afrontar el proceso de hibridación cultural fuertemente presente en la América de la época $-\mathrm{y}$ sobre todo en territorios como Brasil ${ }^{1}$. El Movimiento retoma entonces como metáfora la práctica caníbal de los indios tupys, que era una tribu originaria de la zona. Dicha práctica se llevaba a cabo no como sostén alimenticio, sino bajo la forma de ritual, ya sea como ofrecimiento a los dioses o como manera de adquirir el valor y fuerza del guerrero enemigo. El movimiento modernista retoma esta última acepción en la que, originariamente, no cualquier enemigo era devorado, sino solo los más valerosos, aquellos cuyas cualidades deseaban incorporar a la etnia tupy. Como lo expresa el mismo De Andrade, haciendo un análisis del hecho:

Se trataba de un rito que... expresa un modo de pensar, una visión del mundo que caracterizó cierta fase primitiva de toda la humanidad. Considerada así como una "Weltanschauung" - cosmovisión-, mal se presta a la interpretación materialista e inmoral que de ella hicieron los jesuitas y colonizadores. Al contrario, como acto religioso, pertenece al rico mundo del hombre primitivo. Su sentido armónico y comunal se opone al canibalismo, que viene a ser la antropofagia por gula y

\footnotetext{
${ }^{1}$ Si bien podemos considerar que la presencia y presión de la hibridación cultural a principios de 1900 fue muy marcada y consolidada, no creemos que en la actualidad se haya abandonado completamente el desafio de hacerle frente a dicha presión, aunque sin duda se haya avanzado enormemente en el proceso.
} 
también la antropofagia por hambre ${ }^{2} \ldots$ La operación metafísica ligada al rito antropofágico es la de la transformación del tabú en tótem. Del valor opuesto al valor favorable. La vida es devoración pura (2008, p. 95).

Así, a partir del rito, plantea que todo pasado que les es otro debe ser devorado, pero no sin un criterio de selección que restrinja las opciones posibles, ya que para que una cultura sea admitida como plato en el banquete antropófago no es su sistema de valores per se, ni su lugar en cualquier especie de jerarquía de conocimiento lo que se tiene en cuenta, sino más bien si ese sistema funciona, con qué funciona, y en qué medida proporciona o no medios para crear mundos que respondan a la demanda de una situación determinada. Presenta así una notable embestida contra la recepción acrítica que ensalza a la academia europeizante y postula el devoramiento crítico e irreverente de una alteridad que ha sido siempre múltiple y variable. Buena dosis de irreverencia que mediante la recuperación de tradiciones locales, supone la metabolización propia de lo ingerido con una producción propia como resultado.

El movimiento pretende dar cuenta de la problemática identitaria en la que se encuentra Brasil en ese momento, pero sin que implique por ello la adaptación a un relato nacional. Se inserta entonces en un entramado político-social ya establecido y pretende dar respuesta a las preguntas que se le plantean desde él. De hecho se puede observar que en el transcurso del movimiento hay una modificación de las respuestas que se generan frente al cuestionamiento de una identidad nacional. Primeramente proponen destacar lo propio y realzar lo nacional, patente en el manifiesto "Pau Brazil". Sin embargo, en el Manifiesto Antropófago ya se explicita un corrimiento de esta postura, porque se apartan del planteamiento de una identidad nacional. Si bien se inician en la búsqueda de una respuesta a tal problemática, se alejan de las respuestas estipuladas para proponer una alternativa, algo que genere un cambio en un entramado ya establecido. Incluso pretenden alejarse de la pregunta por una identidad estable, para poder empezar a pensar una identidad múltiple y variable.

Esta propuesta se presenta entonces como un modo de respuesta a la conformación de una determinada ciudadanía, frente a una necesidad concreta en tanto la creación de una "Nación". Encontramos así un movimiento artístico-cultural que es intrínsecamente político, ya que se manifiesta como fundante, o pretende serlo al menos, de otra concepción de ciudadanía sostenida en una concepción también otra de subjetividad (Scherbosky, 2010). Arte-política se presenta así como un núcleo inescindible que articula un proyecto de país alternativo a lo que se venía estableciendo.

Se pretendía ajustar la experiencia brasileña de la vida con la tradición heredada y el modernismo logra hacerlo liberando de manera alegórica -en imágenes y símbolos- datos históricos, sociales y étnicos. Es posible recalcar que, de hecho, buscaban ese "ajuste", ese mixturar sus raíces con una tradición que les había sido

\footnotetext{
${ }^{2}$ Es muy basta la bibliografía acerca del canibalismo, y específicamente en América. Asumimos la distinción clara que marca Oswald de Andrade entre canibalismo y antropofagia. Para ampliar algunas de estas nociones creemos interesante el trabajo de Joaquín Barriendos (2016).
} 
impuesta y no pretendían un regreso al "estado de naturaleza" de los pueblos originarios del Brasil.

La metáfora que eligen no puede ser equiparada a la idea del buen salvaje, ya que además no rechazan -ni en sentido literal ni metafórico- la existencia del conflicto. Rescatan la lucha, pero no como acto de venganza, sino justamente en términos de valoración de la alteridad.

Hay una clara distinción entre lo que este movimiento sostiene como proyecto y las habituales interpretaciones al respecto. Suele pensarse como un proceso de reificación de la alteridad, de actos de violencia desmedida, en definitiva de lo que podemos pensar como prácticas más relacionadas con el canibalismo. No obstante, como aclaramos anteriormente, ya el mismo Oswald de Andrade diferencia estas prácticas en tanto implican concepciones radicalmente distintas. La antropofagia se sostiene en un reconocimiento del otro, lejos de cualquier posibilidad de reificación o fetichización del mismo (aunque siempre está el riesgo de la baja antropofagia).

\section{DEGLUTIR DUALIDADES}

Pensar el proyecto antropofágico como una posibilidad efectiva requiere la visualización de determinados mecanismos que impiden la ruptura y el cambio. Así, para que esta afirmación de la antropofagia se haga efectiva en el presente o al menos llegue a ser una posibilidad real, hay varias cuestiones que se deben tener en cuenta, ya que son parte de los núcleos teóricos que sostienen el proceso. Uno de los puntos que consideramos fundamentales para esta afirmación es la relación categorial centroperiferia. A su vez creemos que dilucidar estos núcleos puede ser un inicio que abra la posibilidad de otro modo de pensar lo estético -y lo político- en Nuestra América.

En primer lugar debemos enmarcarnos dentro del debate que sostienen los Estudios Culturales, ya que de algún modo la antropofagia se da como respuesta a ciertas polaridades binarias como son las nociones centro-periferia, repeticiónmaduración que postula Stuart Hall. En este sentido pensamos la antropofagia como una propuesta superadora, incluso respecto de ciertas versiones de pensadores como Nelly Richard (2009). En efecto, aunque la intelectual chilena se aparta de la posición de Hall y sostiene que no hay una correlación directa entre localización territorial y disposición de los centros hegemónicos sino más bien una red multicentrada, sigue manteniendo las mismas nociones de centro-periferia. Consideramos que Richard marca un primer momento de crítica, ya que genera un inicio de quiebre en esta dualidad tan fija y estable, obviamente sostenida y propagada por el centro. Hay un movimiento global marcado por esta red multicentrada donde encontramos centros en 


\section{Federica Scherbosky}

las periferias y periferias en los centros. Se da como un proceso de la complejidad del mismo Capitalismo Mundial Integrado ${ }^{3}$. Como bien nos señala la autora:

Sabemos que las nuevas formas globales de soberanía capitalista dibujan una cartografía del poder económico-cultural en la que este ya no se agencia desde un foco central sino a través de una red multicentrada. Las segmentaciones dispersas de flujos transversales de esta red multicentrada impiden que "centro" y "periferia" sigan siendo consideradas como localizaciones fijas y polaridades contrarias, rígidamente enfrentados entre sí por antagonismos lineales (Richard, 2009, p. 26).

Junto con esto Richard postula la noción de "función-centro", en el sentido que le da Derrida, para evitar la sobredeterminación topográfica. Aquí vemos, dentro de este primer quiebre, que ya el centro no ocupa un lugar físico fijo, incluso puede ser un "no lugar". Representa simbólicamente la instancia de poder organizar una infinidad de sustituciones de signos, de acuerdo con reglas de poder preestablecidas. No obstante esta modificación y crítica, se trataría en el fondo de mantener la misma lógica, de un acompañamiento "epistemológico" de los cambios que la misma globalización, o como asumimos antes, que el Capitalismo Mundial Integrado va generando. Con la creciente descontextualización de flujos y acontecimientos el capitalismo necesita un cambio en estas nociones que fueron pensadas e impuestas de modo tan estático. El centro y la periferia demandan otro tipo de movilidad simbólica, que es en algún aspecto lo que se logra con esta noción de "función-centro", o con la ubicación de centros en las periferias y viceversa.

Asumimos que el inicio de la complejidad de esta lógica binaria es un avance, ya que empezamos a darnos cuenta que las hegemonías consiguen cooptar las fuerzas de las periferias por un lado, y por otro lado que no todas las manifestaciones periféricas son de por sí alternativas y contrahegemónicas. Esta misma crítica es la que sostiene Nelly Richard (2009) al afirmar que:

las instituciones metropolitanas, que han aprendido a responder a la presión de lo limítrofe y lo excéntrico, diseñan estrategias siempre nuevas de redelimitación de sus fronteras de integración de lo diverso. Tampoco las redes periféricas, por el solo hecho de ser marginales o subalternas a los poderes constituidos, articulan necesariamente significados contrahegemónicos (p. 30).

Creemos entonces, que este podría ser el comienzo de la ruptura de una lógica binaria que no da cuenta de la complejidad real del mundo, y como sosteníamos anteriormente, menos aun de la realidad brasilera. La fusión étnica, cultural, religiosa que presenta no puede reducirse a una lógica binaria, como la que se sigue manteniendo en la teoría del mestizaje por ejemplo. Así, frente al primer

\footnotetext{
${ }^{3}$ Asumimos la noción propuesta por Guattari ya a fines de la década del 70 de "Capitalismo Mundial Integrado" como alternativa a "globalización", ya que no solo le resultaba genérico, sino que sostenía que velaba el sentido fundamentalmente económico, capitalista y neoliberal del fenómeno mundial en su forma actual.
} 
cuestionamiento de estas categorías que produce Richard (2009), la Antropofagia se presenta como una crítica más radical. Si bien acordamos con la autora chilena en que a pesar de la movilidad de las categorías de centros y periferias hay una disparidad en las relaciones de poder que permanece, en tanto;

se ha desimplificado la macrooposición centro/periferia que guiaba emblemáticamente la tradición identitaria del "ser latinoamericano", en su versión anticolonialista y antiimperialista. Pero la dominante capitalista sigue generando asimetrías de poder que reparten desigualitariamente las claves de acceso y participación de lo local en las redes globales de acumulación y transacción del valor semiótico-cultural de todo lo que circula y se intercambia (p. 26).

\section{HACIA UNA DISLOCACIÓN DEL CENTRO}

Estas asimetrías que sostenemos se dan de hecho, es justamente parte de lo que la Antropofagia intenta deglutir, desarticulando la dualidad categorial que rige no solo a nivel discursivo, sino que cala en lo más hondo de la conformación de nuestra subjetividad y a partir de allí en la constitución del mundo en cuanto tal. Ya no más centros ni periferias, sino despliegues a modo de rizoma, como aquello que se amplía incesantemente y se desarrolla sin movimiento fijo.

Encontramos en esta propuesta una dislocación más profunda de la idea de centro, no hay un ataque a la cultura europea pero tampoco una sumisión a ella, ya que cualquiera de las dos posiciones la mantendría como cultura de referencia. Ataque y sumisión serían dos caras de la misma moneda, donde el centro sigue estando fijo y claro, siendo esta estabilidad la que se pretende desarticular. Tampoco se sostiene la posición subalterna, ya que sería una mera inversión de la dualidad. Lo "no europeo" continuaría discriminado como aquello exótico, ya no descalificado, sino enaltecido. Sería, en definitiva, desconocer la fuerza de la afirmación de la mixtura antropofágica, donde no hay jerarquías culturales a priori y donde todos los repertorios son pasibles de ser deglutidos en esta búsqueda de recursos para crear cartografías de sentido.

Sin embargo, estos repertorios no son tomados como sistemas en su totalidad, si no que se absorben aquellos elementos que permiten transmitir determinadas intensidades de sentido de un sistema, otras de otro y así se alejan de cualquier connotación identitaria, de cualquier sistema acabado y completo. Buscan una mixtura que se potencie sin límites.

Es admisible recalcar, como ya lo hicimos anteriormente, que hay que asumir con cuidado esta deglución de dualidades y jerarquías. En lo que respecta a las dualidades es la propuesta de base de la antropofagia. No obstante, diluir las jerarquías podría llevar a solapar las desigualdades existentes, hecho que sería pensable como aquella llamada "baja antropofagia", como la desvirtuación posible y a la vez intrínseca que este movimiento presenta. Consideramos de igual modo que vale la pena el riesgo y que al menos no hay una intención clara de solapar desigualdades, pues hay un ideario emancipador en la 


\section{Federica Scherbosky}

antropofagia, que pretende llegar a esa instancia donde el diluir las jerarquías no sea solaparlas -aunque esto se presenta casi a modo de utopía-. De igual manera sí es necesario señalar que la mixtura antropofágica deglute jerarquías de cualquier tipo, pues no están establecidas de modo jerárquico justamente. No hay un a priori que las ordene, por lo que absorben lo que necesitan de cada una para su mixtura singular.

Si volvemos al contexto de surgimiento de la Antropofagia podemos remarcar que la cartografía europea no era viable en la cultura brasilera. Se agrega además que la cultura europea y burguesa ya había entrado en crisis con la Primer Guerra Mundial. Así, por esos años la reivindicación antropofágica se presentaba como una línea de fuga de la cultura europea. No más oposición, ni referencia, ni centro. "El árbol del saber occidental trasplantado a la América tropical deja de ser árbol, no tiene sentido su estructura y evolución previamente definidas por un programa trascendental"4 (Rolnik, 1998, p.138). A diferencia de esto, se reivindica un proceso infinito de creación, con diferentes referencias y recursos que genera una evolución que se puede redefinir permanentemente. Podemos pensar entonces que este movimiento se dio de manera rizomática, como aquello que se extendió de modo imprevisible y caótico por toda la cultura local.

\section{COSMOPOLITISMO ESTÉTICO O DE UN NUEVO SISTEMA COLONIAL}

En el sistema colonial se articularon ciertas jerarquías estético-geopolíticas que posibilitaron que un entramado particular permaneciera como universal, fijo, estable y sobre todo "natural". Hay un uso coercitivo del pensamiento geográfico puesto al servicio de una construcción territorial de las identidades sumamente racializante y una política de traducción de las culturas bajo la misma tónica.

El discurso poscolonial cree ver en las turbulencias culturales actuales una superación de las estructuras modernas que llevarían a un cosmopolitismo estético, a un crisol cultural -bastante mezclado con las ideas del crisol de razas típicas de los discursos multiculturalistas-. Esto indica que las estrategias de agenciamiento hegemónicas siguen en pie y mantienen el mismo sistema con algunas reformas. Solo que estas supuestas reformas generan una mayor flexibilidad y desapego del territorio "material" en cuanto tal, y una dispersión de este agenciamiento mediante las políticas de subjetivación diseñadas o pensadas para gestiones transculturales. Cuestión que señala muy bien Suely Rolnik con su noción de "identidades prêt-à-porter", consecuencia de una flexibilidad en la constitución misma de la subjetividad antropofágica y que remarca, en relación con la alteridad, Joaquín Barriendos (2011), quien señala que:

El nuevo internacionalismo del arte contemporáneo —en el que se pretende que aparezcan todas las culturas 'estéticamente' bien representadas - extiende la

\footnotetext{
${ }^{4}$ Es importante resaltar aquí la utilización de la metáfora del árbol, ya que Deleuze opone a la forma de árbol el rizoma. Frente a una planta determinada con un modo específico de desarrollo y crecimiento postula la expansión imprevisible e infinita del rizoma.
} 
polaridad etnocéntrica de la modernidad hasta tal éxtasis (hasta una 'sobreidentificación' con el otro tan extrema) que la alteridad se convierte en su interior en algo geopolíticamente estéril; tan antropologizable como antropolarizado. Es por esta razón por la que Olu Oguibe ha llamado monolíticas a estas tendencias internacionalistas del arte contemporáneo. Esta reaparición simbólica del otro conlleva no solo la visualización de la alteridad en tanto que sujeto subalterno representable, sino también la banalización del conflicto mismo de la alteridad, su estetización como fetiche en un mundo en el que lo periférico, lo híbrido y lo subalterno se han vuelto obscenamente cotidianos (en línea).

Podemos pensar aquí en la tensión o dualidad que plantea Grüner acerca de la estetización de la política o la politización del arte al retomar el conocido texto de Benjamin "La obra de arte en la época de la reproductibilidad técnica". Sin dudas el autor reivindica la politización del arte, pero entendiendo este proceso en un sentido amplio. Mientras que sostiene que en las distintas políticas fascistas se presenta claramente una estetización de la política, en términos más acotados -como es el caso de los fascismos- o en un aspecto más amplio -como podría pensarse en la actualidad la reivindicación de opciones multiculturalistas- que perpetúan el statu quo, fetichizando al otro y anulando así cualquier posibilidad de cambio o acontecimiento.

En este aspecto el movimiento antropofágico se puede pensar como una alternativa válida en tanto busca dar una respuesta no solo a la fetichización del otro, reivindicándolo en su alteridad y revalorizando sus atributos y fortalezas, sino que además rescata el conflicto como ámbito de confrontación, aprendizaje y crecimiento. Es posible pensar además que lo periférico, lo subalterno y lo híbrido se han vuelto obscenamente cotidianos en términos de funcionalidad a este sistema-mundo, donde además la posibilidad de representación real muchas veces es negada. Es quien se erige como centro el que otorga visibilidad a estos otros, siendo esta una determinada visibilidad. Pensamos aquí en toda la literatura denunciante de los estudios subalternos que perfectamente hace hincapié en la posibilidad de autorrepresentarse, de conformar una subjetividad propia y de crear una normativa estético-cultural adaptada a la realidad en la que viven ${ }^{5}$.

En esta cotidianidad de lo periférico las fronteras pasan a ser entidades centrales para la comprensión del mundo actual y se da una "recentralización" de la periferia, sobre todo en términos simbólicos, que es lo que patentiza Nelly Richard con su noción de "función centro". Como señala acertadamente Joaquín Barriendos (2011):

entre la búsqueda de visibilidad de lo subalterno para reposicionarse y reorientar su relación frente al mainstream y las necesidades de apropiación e internacionalización occidentalista de la alteridad para volver coherente el discurso poscolonial ha surgido un evidente conflicto de intereses, el cual se

\footnotetext{
${ }^{5}$ El contundente libro de Franz Fanon, Piel negra, máscaras blancas da cuenta de este complejo proceso y aboga justamente por la posibilidad de liberar la propia capacidad de representación, de palabra, de conformación de uno mismo.
} 
manifiesta en el arte contemporáneo por medio de la estetización de lo fronterizo y de la 'defensa' neopaternalista de lo marginal. En la actualidad asistimos por lo tanto a un inexpugnable aprovechamiento estético del subdesarrollo. Esta plusvalía estética agenciable es la que está en juego en los procesos de exotización, internacionalización y comercialización del arte contemporáneo (en línea).

Ha habido un traslado desde la imagen de la abundancia que atrajo a las culturas del descubrimiento, hacia un axioma estético de la austeridad y la carencia. Esto es lo que Barriendos llama el "activo periferia" dentro del sistema internacional del arte contemporáneo, es la función económica de las periferias estratégicas.

FETICHIZACIÓN DE LA ALTERIDAD O DE LA APROPIACIÓN ESTRATÉGICA DE LA PERIFERIA

Podemos observar entonces dos planos de análisis diferenciados en este complejo entramado de modernidad-colonialidad-conformación de la subjetividad y políticas estéticas. Uno el que analiza Barriendos en un plano fáctico, de apropiación de la diferencia y estetización de la misma; y otro podría ser justamente aquel punto de vista que señale la potencialidad estética en esta perspectiva de colonialidad que genera un paradigma multiculturalista de aparente coexistencia y tolerancia con la diferencia, cuando en realidad no hay más que exaltación de la misma. Una buena folklorización de lo exótico.

Hay un aprovechamiento estético del subdesarrollo que conlleva esta folklorización y mercantilización de la cultura. Esta reificación se sustenta en una visión romántica de lo primitivo, donde se piensa que fuera de Occidente los artistas tienen más contacto con el ser natural, con lo primitivo, con la vida en estado puro, con la masa, con el pueblo, con la naturaleza ${ }^{6}$. Actitud que implica tanto una exotización de la alteridad como así también una suposición del arte como mímesis. $\mathrm{O}$ en otras situaciones supone pensar el arte político solo como aquel explícitamente político, que implicaría una "verdadera" reivindicación del pueblo, de la masa, de los trabajadores, etc. Actitudes que consideramos no solo simplistas sin más, sino sumamente funcionales a esta lógica de reificación y fetichización de la alteridad.

En una segunda dimensión se puede pensar la potencialidad del arte como la posibilidad de un reconocimiento desde otro ámbito, como aquello que al surgir de manera lindante al modo de racionalidad colonial, puede justamente cuestionarla, o cuando menos puede haber un cierto movimiento, una distancia que habilite la crítica y una posible apertura en este sistema aparentemente cerrado y funcional ${ }^{7}$.

Consideramos, de igual modo, que más allá de las críticas y corrimientos/quiebres producidos hasta ahora, sigue existiendo un proceso de desautorización en la

\footnotetext{
${ }^{6}$ Podría pensarse en Gaugin y sus viajes, en el afán de encontrarse con "la naturaleza".

${ }^{7}$ Esto puede ser desarrollado ampliamente a partir de la propuesta de la Escuela de Frankfurt, ya que postula al arte como alternativa crítica frente a un proceso de modernidad (colonialidad, decimos nosotros) sostenida en la razón instrumental.
} 
internacionalización del arte, que desvaloriza $\mathrm{u}$ occidentaliza $\mathrm{y}$ recentra a las epistemologías y estéticas periféricas. Vale la pregunta de Barriendos por una curaduría de la diversidad cultural que pueda correrse de esta exotización de la diferencia, que no hace más que generar desigualdad. Es la pregunta y la búsqueda de otro modo de percepción, que rompa el círculo de la mirada occidental sobre lo "no curado". Pregunta a la que no se puede dar respuesta hasta que no empiece a desarticularse la matriz colonial y la occidentalización geopolítica de las subjetividades modernas. Habrá que empezar a recartografiar al sujeto, a aquel sujeto moderno, autónomo, libre y universal, que se pasea por escenarios multiculturales confortablemente. Quizás la antropofagia es un buen modo de empezar a recartografiarlo, a desandar algunos trayectos y emprender nuevos caminos que posibiliten un redescubrimiento estético. Propuesta que invita a deconstruir las jerarquías establecidas en relación sobre todo a este "arte periférico", que lejos de posibilitar una apertura y una verdadera política de reconocimiento se sostiene en una aparente integración que no hace más que reificar a la alteridad. Se produce una apropiación estratégica de esta "periferia" que sin dudas refuncionaliza el sistema.

\section{ACTIVO PERIFERIA O ACERCA DE LA EXOTIZACIÓN DE LA ALTERIDAD}

Así como la flexibilidad que suscitó la antropofagia fue cooptada por el neoliberalismo, posibilitando una nueva reconversión de sí, una nueva expansión y consolidación, creemos que con la dualidad centro-periferia sucede algo similar. Este es el "activo periferia" del que habla Barriendos, una utilización de la periferia, de la alteridad, de aquello considerado como exótico en función de una geopolítica estética determinada. Consideramos de hecho que hay más posibilidades de caer en la baja antropofagia, ya que hay una mayor tendencia a reificar la alteridad, a fetichizar la periferia y exotizarla en tanto se perpetúe este modo de percibir que fija de manera estanca centros y periferias.

$\mathrm{Al}$ igual que en la baja antropofagia se genera una instrumentalización al servicio de determinados intereses, ya que la alteridad es incorporada justamente como instrumento. No hay un deseo real del otro, en tanto amplía el horizonte personal de sentido.

Según Rolnik frente a la sociedad disciplinaria se reactiva la experiencia antropofágica en un movimiento de contracultura que se condensa en el "tropicalismo", que desborda el territorio delimitado donde se enmarcaban las vanguardias artísticas. Cabría pensar en este paso de las sociedades disciplinarias a las sociedades de control -según la clasificación que hace Deleuze- en el modo en que podría resurgir la experiencia antropofágica. Quizás sea una instancia para rever las categorías con las que pensamos las experiencias estéticas latinoamericanas y a partir de allí pueda producirse este desfondamiento de las mismas, ya que son categorías que impiden el encuentro arte-política (resistencia-creación).

Concordamos con la autora brasilera en que la alternativa posible vendrá al aunarse "resistencia y creación". El proceso va de manera conjunta. No obstante, como 
ya señalamos, el Capitalismo Mundial Integrado fomenta la creación pero separada de la resistencia, se despotencia esta parte fundamental del proceso y se la vacía. Así, esta creación apropiada es llevada hacia las esferas del mercado, produciendo sin cesar mundos-mercancía e identidades prêt-à-porter. Ejemplo de esto es cómo en Brasil en los 60 se dan de manera escindida los movimientos de resistencia política y los de resistencia cultural. El núcleo arte-política se había quebrado, porque era justamente ahí donde podía empezar a pensarse una potencialidad alternativa.

Consideramos necesario repensar la propuesta de la subjetividad antropofágica que rehabilita la creación para la vida, pues la política y el arte son las zonas en las que se puede desobstruir el cuerpo vibrátil ${ }^{8}$. Una zona híbrida conformada conjuntamente, donde los límites son difusos, donde se contaminan uno con el otro a causa de su vecindad. El arte a partir de la creación y la política a partir de la resistencia potenciarían esta zona híbrida volviendo a reunir lo que es parte de un mismo proceso. La práctica artística reactualiza las sensaciones con aquella presencia de la alteridad que modifica las formas existentes. Se posiciona así como un medio para dar a conocer la nueva disposición de fuerzas que afectan a una sociedad. Por esto la creación como fuerza se encuentra en todo el campo social, ya no relegada al campo específico del arte. Insistimos entonces en que si la creación permanece exclusivamente en el campo artístico pierde conexión con la fuerza de resistencia y pasa a ser, una vez más, fuente de valor del capital, que genera objetos artísticos pasibles de ser consumidos sin cesar. Como bien nos decía Suely Rolnik pasan a ser objetos artísticos cargados de glamur que funcionan a modo de droga de estas identidades prêt-à-porter. Identidades comercializables en función de las mercancías culturales que las conforman. La disociación del producto artístico respecto del proceso de producción se hace cada vez más notoria. El plus valor de acuerdo con el logotipo o lo que el mercado demande vuelve al sistema cada vez más perverso y a la subjetividad cada vez más alejada del cuerpo vibrátil.

La propuesta frente a esta situación es la apertura de la creación a todo el campo social, ya que es el modo de hacer patente la composición de fuerzas por las que nos vemos afectados. Se busca la reunificación de creación y resistencia para poder asumir la presencia de una alteridad no fetichizada. Sin embargo, si sigue escindida la resistencia de la creación, esta última permanecerá cooptada por el Capitalismo Mundial Integrado, haciendo de la creación un proceso de producción de mercancía que mercantiliza a la misma capacidad de creación y fetichiza a la alteridad. Esto es parte de una determinada geopolítica estética que perpetúa determinadas categorías -como en

\footnotetext{
${ }^{8}$ La noción de cuerpo vibrátil es propuesta y analizada Suely Rolnik (2006) en su libro Cartografia Sentimental. Transformaciones contemporáneas del deseo, en 1989. No obstante la sigue manteniendo y desarrollando en el resto de sus escritos, haciendo referencia al cuerpo vibrátil como aquello que en el cuerpo es susceptible de ser afectado por fuerzas del mundo y que no depende de su condición de orgánico, de sensible, de erógeno sino de carne recorrida por ondas nerviosas (Rolnik, 2005, 2006).
} 
este caso analizamos la de centro y periferia- para poder hacer uso de la alteridad enmarcada en la periferia y reinventar cada vez nuevas mercancías y nuevos mercados. Esta situación es la reutilización de la periferia que denuncia Joaquín Barriendos al proponer pensar el "activo periferia".

Por esto es que nos resulta importante pensar estos procesos a partir de una modificación en el concepto mismo de subjetividad, que nos lleve a otro modo de relacionarnos con la alteridad, a otro estar juntos. A partir de esta nueva subjetividad que asume una nueva concepción de lo artístico como modo de actualizar la presencia viva del otro es que podría empezar a pensarse en una nueva propuesta categorial que abandone la dualidad centro-periferia.

\author{
Universidad Nacional de Cuyo* \\ Becaria posdoctoral de INCIHUSA - CONICET \\ Av. Ruiz Leal s/n Parque Gral San Martin. Mendoza (Argentina) \\ fedescherbo@yahoo.com.ar
}

\title{
OBRAS CITADAS
}

Barriendos, Joaquín. (2016). Apetitos extremos: La colonialidad del ver y las imágenesarchivo sobre el canibalismo de Indias. Disponible en: http://eipcp.net/ transversal/0708/barriendos/es (4/02/2016)

— (2011). Desconquistas (políticas) y redescubrimientos (estéticos). Disponible en: $\mathrm{http}: / / w w w . d e s-b o r d e s . n e t / d e s-b o r d e s / j o a q u i n$ barriendos.php (10/11/2011)

De Andrade, (2008). Oswald. Escritos Antropófagos. Buenos Aires. Ediciones Corregidor.

Richard, Nelly. (2009). En torno a la noción de "Sur". En: Revista Ramona nº 91. Fundación Start. Buenos Aires.

Rolnik, Suely. (2006a). Cartografia Sentimental. Transformações contemporâneas do desejo. Porto Alegre: Sulina.

— (2006b). Geopolítica del rufián (o del chuleo, o del cafishio) En: Revista Ramona 67. Disponible en: http://www.ramona.org.ar/ Consultado el 24/07/2010.

— (1998). "Subjetividade Antropofágica/Anthropophagic Subjectivity". En: Paulo Herkenhoff \& Adriano Pedrosa (Edit.). Arte Contemporânea Brasileira: Um elentre Outro/s, XXIV ${ }^{a}$ Bienal Internacional de São Paulo. São Paulo: Fundación Bienal de São Paulo, 128-147. Edición bilingüe (portugués/inglés).

Scherbosky, Federica. (2010). "La subjetividad antropofágica: aportes para una concepción devorativa de la vida." Estudios de filosofia práctica e historia de las ideas. [online]. Vol.12, №2, 23-31. 